\title{
Diagnostic utility of an immunochromatography test for the detection of Leptospira IgM antibodies in domestic dogs ${ }^{1}$
}

\author{
Lucía Azócar-Aedo ${ }^{2 *}$, Henk Smits ${ }^{3}$ and Gustavo Monti ${ }^{4}$
}

\begin{abstract}
Azócar-Aedo L., Smits H. \& Monti G. 2017. Diagnostic utility of an immunochromatography test for the detection of Leptospira IgM antibodies in domestic dogs. Pesquisa Veterinaria Brasileira 37(7):708-712. Facultad de Ciencias Veterinarias, Universidad Austral de Chile, Independencia 641, PO Box 567, Valdivia, Chile. E-mail: luciaazocaraedo@gmail.com

A cross-sectional study using 99 serum samples of dogs from southern Chile was conducted to determine the diagnostic utility of a rapid immunochromatography assay for the detection of Leptospira specific IgM antibodies as screening test and as a potential aid in the diagnosis of leptospirosis in animals with and without clinical suspicion of the disease. The Microscopic Agglutination Test (MAT) was used as reference assay. Anti-Leptospira antibodies were detected in $37.3 \%$ of the dogs with MAT. Using the immunochromatography test, specific IgM antibodies were found in $13.1 \%$ of sampled dogs. The sensitivity of the rapid test as screening assay was $29.7 \%$ (95\% Confidence Interval=16.4-47.2) and the specificity was $96.7 \%$ (95\% Confidence Interval=87.8-99.4). $40.0 \%$ of the canines with clinical suspicion of leptospirosis and $37.1 \%$ of dogs without clinical signs were serological reactors to MAT, but none of MAT reactive dogs with clinical suspicion tested positive in the rapid test. Rapid and user-friendly diagnostic procedures for canine leptospirosis such as this immunochromatography assay could be important tools to use in clinical practice, however, further studies are needed to obtain more information about their utility, considering that diagnostic tests could not have similar performances in different geographic locations, clinical and epidemiological contexts.
\end{abstract}

INDEX TERMS: Leptospirosis, dogs, rapid immunochromatography test, Microscopic Agglutination Test, diagnostic utility.

\section{INTRODUCTION}

Leptospirosis is the most widespread zoonotic disease in the world, caused by the infection with a motile spirochaetal bacterium of the genus Leptospira (Bharti et al. 2003). In the last years, a re-emergence of leptospiral infection in pet populations in some geographic areas in the world have raised the concern about the disease (Damborg et al. 2015, Major et al. 2014). However, the understanding of their epidemiology and pathogenesis still is limited (Schuller et al. 2015).

\footnotetext{
${ }^{1}$ Received on November 2, 2015.

Accepted for publication on June 25, 2016.

${ }^{2}$ Facultad de Ciencias Veterinarias, Universidad Austral de Chile, Independencia 641, PO Box 567, Valdivia, Chile. ${ }^{*}$ Corresponding author: luciaazocaraedo@gmail.com

${ }^{3}$ Kit Biomedical Research, Royal Tropical Institute 63, 1092 AD Amsterdam, Netherlands.

${ }^{4}$ Instituto de Medicina Veterinaria Preventiva, Facultad de Ciencias Veterinarias, Universidad Austral de Chile, Independencia 641, PO Box 567, Valdivia, Chile.
}

When suspected cases of leptospirosis are present in canine clinical practice, a diagnosis is essential because an early treatment is important to ensure the recovery of the patient (Dey et al. 2007) Clinical features in dogs vary from subclinical infections to multiorganic involment with renal, hepatic, hematologic and pulmonary failure (Guerra 2009, Sykes et al. 2011), therefore specific diagnostic testing is required to confirm a diagnosis (Bolin 1996).

MAT remains the standard serological test for canine leptospirosis and positive titres confirm the exposure of the animal to the bacterium (Sessions \& Greene 2004, Gaschen 2008). Despite this, there is no consensus about the definitive diagnostic titre (Bolin 1996). In addition, MAT is a time-consuming assay, particularly when the panel of serovars used is large, it only can be performed in well-equipped laboratories and the cost of the Leptospira strain maintenance could be high, make it difficult to use as a routine diagnostic method (Faine 1994, Safiullah et al. 2009). 
There is a lack of awareness of leptospirosis mainly due to the subclinical nature of the disease and the technically demanding laboratory tests as MAT, making the disease difficult to diagnose both in the clinic and in the laboratory (WHO 2001, Hartskeerl 2003, Vieira et al. 2006). These issues contribute to the process: under-diagnosis, under-reporting and neglection of the disease (Hartskeerl et al. 2011).

Recently, a simple and rapid immunochromatographic lateral flow assay device detecting Leptospira specific IgM antibodies in domestic dogs was developed. The assay may be used by the veterinary practitioner without the need for special laboratory equipment and experience and provides an almost instantaneous result (Abdoel et al. 2011).

The aim of this study was to assess the diagnostic utility of the rapid immunochromatographic assay as screening test and as a potential aid in the diagnosis of leptospirosis in a group of dogs with and without clinical suspicion of this disease.

\section{MATERIALS AND METHODS}

Ethics statement. The study protocol was approved by the bioethics committee at the Universidad Austral de Chile, Valdivia, Chile (certification \#10-2012).

Study design. Between January and September 2011, a cross-sectional study was performed on 99 domestic dogs of different breeds, older than two months of age from the cities of Valdivia, Osorno, Paillaco, San Pablo (urban areas) and from dairy farms (rural areas) in Los Rios and Los Lagos regions in southern Chile. The sample size was 99 dogs because that number of test devices of the immunochromatography assay was available.

Patients attending private veterinary clinics, canines in a veterinary neutering campaign and dogs during home and farm visits were enrolled. Blood samples $(1-2 \mathrm{ml})$ were extracted by venipuncture. The owners voluntarily agreed to participate in the survey.

Microscopic agglutination test (MAT). The MAT was executed with live cultures of six reference strains representative of Leptospira interrrogans serovars Hardjo, Pomona, Canicola, Icterohaemorragiae and Autumnalis and L. borgpetersenii serovar Ballum that occur in animals in Chile (Riedemann \& Zamora 1987). The reference strains for each serovar were Hardjoprajitno (Hardjo), Pomona (Pomona), Hond Utrecht IV (Canicola), Verdun (Icterohaemorragiae), Akiyami A (Autumnalis) and S102 (Ballum) and it were cultured in the Ellinghausen-McCullough-Johnson-Harris medium.

The MAT was performed in tubes on 2-fold serial serum dilutions from 1:100 to 1:1.600 following standard recommendations (WHO 2003).

The criteria for considering a sample with anti-Leptospira antibodies in MAT were the following:

i) For unvaccinated dogs, a single titre of 1:100 or greater in the diagnostic test (Alton et al. 2009).

ii) Dogs develop relatively low antibody titres in response to vaccination, mostly $1: 100$ to $1: 400$ and it may persist at these levels for 1 to 3 months after vaccination (Bolin 1996, Greene et al. 2006, Van de Maele et al. 2008), therefore, the antitibody titres and the time since vaccination were considered to classify the dogs as serological reactors to MAT. For animals vaccinated from 1 to 3 months before the collection of the blood sample, titres of 1:400 or higher were considered as positives and for dogs vaccinated 3 to 12 months before sampling, titres of 1:200 or higher were considered as positives. The vaccine most commonly used in Chile contains Leptospira interrogans serovars Canicola and Icterohaemorrhagiae. Other vaccines may contain L. interrogans serovar Pomona and L. kirschneri serovar Grippotyphosa.

iii) For dogs reacting positively in MAT for more than one serovar, the serovar with the highest titre was specified as the cause of the serological reaction to Leptospira and reactions to different serovars at the same titre were considered coagglutinations (Silva and Riedemann 2007).

Rapid canine leptospirosis test. This rapid immunochromatography test can detect specific IgM antibodies to Leptospira pathogenic serovars (Abdoel et al. 2011). The test is a lateral flow assay and consists of a plastic device containing a composite assay strip with a test and a control line and includes a colored detection reagent that allows the visual detection of specific IgM antibodies bound at the test line. The test line consists of a Leptospira specific antigen derived from the pathogenic leptospiral strain Wijnberg of L. interrogans serovar Copenhageni and the detection reagent consists of an antidog IgM antibody conjugated to $40 \mathrm{~nm}$ colloidal gold particles.

The rapid test was performed according to the designer's recommendations by adding $5 \mu \mathrm{l}$ of serum to the sample pad followed by the immediate addition of $130 \mu \mathrm{l}$ of running fluid (PBS containing $1.67 \%$ bovine serum albumin and $3 \%$ Tween 20 ). The result was read after 10 minutes by visual inspection. A valid result was obtained if staining of the control line was observed. When a staining on the test line was noted, the sample was considered positive for the presence of Leptospira specific IgM antibodies.

Determination of the presence of clinical signs of leptospirosis. All animals were subject to a physical examination by a veterinarian to determine their health status. Given the variability of clinical presentations of canine leptospirosis (Langston \& Heuter 2003, Sykes et al. 2011, Schuller et al. 2015), the possible clinical signs present in the dogs were considered as "primary" or "secondary" (Table 1) and the animals were classified as with "clinical suspicion of leptospirosis" if they met the following criteria: dogs that had at least one primary clinical sign with or without one secondary clinical sign.

Evaluation of the rapid immunochromatography assay as screening test. The performance of the rapid test as screening assay was assessed using MAT as reference test. The sensitivity (Se) and specificity (Sp) were calculated based in a published methodology (Dey et al. 2007). Confidence intervals of 95\% (95\% CI) were obtained with EpiInfo version 6.04.

\section{RESULTS}

Serovars and antibody titers. Anti-Leptospira antibodies were detected with MAT in 37/99 (37.3\%) of the dogs. The most frequent serovars were $L$. interrogans serovar Canicola $(24.3 \%)$ and $L$. borgpetersenii serovar Ballum (18.9\%), followed by L. interrogans serovars Pomona (16.2\%), Autumnalis (13.5\%), Icterohaemorragiae (10.8\%) and Hardjo (5.4\%), which belongs to serogroups Canicola, Ballum, Pomona, Autumnalis, Icterohaemorragiae and Sejroe, respectively. In addition, there were four co-agglutinations with Canicola and Icterohaemorragiae (2.7\%), Canicola and Hardjo (2.7\%), Ballum and Pomona (2.7\%), and Ballum and Autumnalis (2.7\%). The antibody titers ranged between 1:100 and 1:1600 and the most frequent levels were 1:100 and 1:200. 
Diagnostic utility of the rapid immunochromatography test as screening assay. Using the rapid immunochromatography test, Leptospira specific IgM antibodies were found in 13/99 (13.1\%) sampled animals. The estimated Se of the immunochromatography test relative to MAT was $29.7 \%(95 \% \mathrm{CI}=16.4-47.2)$ and the Specificity was $96.7 \%$ (95\% CI=87.8-99.4\%)

Diagnostic utility of the immunochromatography test as a potential aid in the diagnosis of leptospirosis. Physical examination identified 10 dogs with suspicion of leptospirosis according their clinical signs, and 89 animals without clinical suspicion of the disease. Using MAT, the frequency of anti-Leptospira antibodies for dogs with clinical suspicion of leptospirosis was $40.0 \%$ and for dogs without clinical suspicion was $37.1 \%$ (Table 2). MAT titres ranged from 1:100 to 1:800 for the 4 dogs with clinical suspicion of leptospirosis, which agglutinated at the indicated titres with serovars Autumnalis (1:200), Ballum (1:800), Canicola (1:100) and Pomona (1:200). Antibody titres for the 33 seropositive dogs without clinical signs ranged from 1:100 to $1: 1600$.

None of the dogs with clinical suspicion of leptospirosis tested positive with the rapid immunochromatography test. However, specific IgM antibodies with this assay were detected in $13(14.6 \%)$ of the animals without clinical signs of the disease (Table 2).

The information obtained from dog owners revealed that 15 dogs without clinical suspicion of leptospirosis had a documented history of vaccination with the bivalent vaccine containing L. interrogans serovars Canicola and Icterohaemorrhagiae. Using MAT, serological reactors were found in $3(20.0 \%)$ vaccinated and in $30(40.5 \%)$ unvaccinated

\section{Table 1. Primary and secondary clinical signs of leptospirosis}

\section{Primary clinical signs}

Depression, anorexia, fever, icterus, renal alterations in the biochemical profile (increased serum urea and/or creatinin), hepatic alterations in the biochemical profile (increased serum alanine aminotransferase, aspartate aminotransferase and/or alkaline phosphatase).

2. Secondary clinical signs:

Vomiting, polyuria, polydipsia, muscle pain, reluctance to movements, diarrhea, abdominal pain, renomegaly, hepatic sensitivity, petechiae and/or ecchymosis in buccal mucous membranes, dyspnea and cough, dehydration, lymphadenopathy.

Table 2. MAT and rapid immunochromatography test results according to vaccine status and clinical suspicion of leptospirosis

\begin{tabular}{lccccc}
\hline Group and subgroup & \multicolumn{3}{c}{$\begin{array}{l}\text { No. (\%) of dogs with the following results } \\
\text { for the detection of Leptospira antibodies }\end{array}$} \\
\cline { 2 - 3 } \cline { 2 - 3 } & \multicolumn{2}{c}{ MAT } & & \multicolumn{2}{c}{ Rapid test } \\
\cline { 2 - 3 } \cline { 5 - 6 } & Positive & Negative & & Positive & Negative \\
\hline $\begin{array}{l}\text { Dogs with clinical suspicion } \\
\text { of leptospirosis (n=10) }\end{array}$ & $4(40.0)$ & $6(60.0)$ & & $0(0)$ & $10(100)$ \\
$\begin{array}{l}\text { Vaccinated (n=0) } \\
\text { Unvaccinated (n=10) }\end{array}$ & $4(40.0)$ & $6(60.0)$ & & $0(0)$ & $10(100)$ \\
$\begin{array}{l}\text { Dogs without clinical of sus- } \\
\text { picion leptospirosis (n=89) }\end{array}$ & $33(37.1)$ & $56(62.9)$ & $13(14.6)$ & $76(85.4)$ \\
$\begin{array}{l}\text { Vaccinated (n=15) } \\
\text { Unvaccinated (n=74) }\end{array}$ & $3(20.0)$ & $12(80.0)$ & $7(46.7)$ & $8(53.3)$ \\
& $30(40.5)$ & $44(59.5)$ & $6(8.1)$ & $68(91.9)$
\end{tabular}

dogs. With the immunochromatography assay, 7 (46.7\%) samples from vaccinated dogs and $6(8.1 \%)$ samples from unvaccinated dogs tested positive (Table 2).

\section{DISCUSSION}

Using MAT, seropositivity to L. interrogans serovars Canicola, Pomona, Autumnalis, Icterohaemorragiae, Hardjo and $L$. borgpetersenii serovar Ballum was diagnosed in domestic dogs in this study. Serovars Canicola and Ballum were detected frequently, which coincides with earlier surveys conducted in Chile (Silva \& Riedemann 2007, Lelu et al. 2015). Exposure of animals to specific Leptospira serovars is determined by different factors including the presence of specific reservoir hosts in the geographic area (Acha \& Szyfes 2003). For serovars Canicola and Ballum, the primary reservoir hosts are dogs and mice, respectively (Greene et al. 2006). Serovar Icterohaemorragiae is associated with rats; cattle and sheep may harbor serovars Hardjo and Pomona, while pigs may harbor Pomona (Bolin 2000). This provides information about the potential origin of the infection and it could help to take prevention measures for avoiding the exposure of the dogs to the bacterium.

Out of the reactive serovars, two of these: Leptospira interrogans serovars Canicola and Icterohaemorragiae are included in the most commonly used vaccines in Chile. Other existing vaccines contain L. interrogans serovar Pomona and L. kirschneri serovar Grippotyphosa. Vaccination for leptospirosis does not provide cross-protection to other serovars and the detection of pathogenic leptospiral serovars not included in the available vaccines such as Autumnalis and Hardjo raises the concern about the expediency of vaccination and the risk of infection. If infection of dogs with these serovars can be confirmed by bacteriological culture and proven to be associated with severe disease or if it can be transmitted to the people, dogs can act as sentinels for human infections and further considerations of the effectiveness of the current vaccination practices are needed. Just 15 of the dogs included in the study had been vaccinated against Leptospira infection (Table 2); therefore a public awareness campaign may be necessary to promote vaccination.

MAT can reveal serological reactions for different Leptospira serogroups (Picardeau 2013). Despite this, leptospiral antibodies may not be detectable when a representative strain for a serogroup is not represented in the panel used (Safiullah et al. 2009). Since in this study the MAT panel was restricted to 6 serovars, it is possible that some dogs with anti-Leptospira antibodies were not detected.

The estimated Se of the rapid immunochromatography test as screening assay was $29.7 \%$. The diagnostic utility of this test was evaluated elsewhere in samples of dogs from The Netherlands and the Se was 100\% (Abdoel et al. 2011). According to Greiner \& Gardner (2000), the Se in serologic tests is variable depending on the infection phase and the immune status of the patient and these factors varies over time. Regarding this, infection with pathogenic Leptospira produces a quick rise of specific serologic IgM antibodies during the acute phase, followed by the development of serovar-specific agglutinating antibodies (Greene 
et al. 2006). For these reason, the detection of specific IgM Leptospira antibodies with the immunochromatography test can be more suitable in acute cases of disease. Considering that MAT identifies IgM and IgG antibodies (Faine 1994) and the titers observed in MAT serological reactive dogs in the present study mostly were low and paired serum samples to diagnose current infections were not used, it is possible that some serological reactions were ancient infections with an immune response with predominance of IgG antibodies, which were not detected with the rapid test and it could explain the different Se regarding the study in The Netherlands.

On the other hand, the estimated Sp of the immunchromatography test was high (96.7\%), which is consistent with a previous study (Abdoel et al. 2011). Diagnostic tests with high $\mathrm{Sp}$ in leptospirosis could be useful when is not possible to obtain paired serum samples to confirm the diagnosis with MAT. Additionally, the immunochromatography test could be a suitable alternative to use when other diagnostic assays are not available.

In this study, only 10 dogs with clinical suspicion of leptospirosis were found and the application of the immunochromatography assay gave a negative result for all these animals (Table 2). The antigen used in this test is the local strain Wijnberg of L. interrogans serovar Copenhageni from The Netherlands (Abdoel et al. 2014). Although this antigen can contain serogroup and broadly reactive Leptospira genus specific fractions (Terpstra et al. 1985), the prevalence of enzootic leptospiral serogroups differ geographically and the reactivity with this antigen may be lower with other serogroups, which could explain the negative results of the rapid test.

Out of 10 dogs with clinical suspicion of leptospirosis, 4 gave a positive result to MAT. According to Greene et al. (2006), after natural infections, MAT titers become positive approximately after 1 week of infection, with a peak at 3 to 4 weeks and it could remain positive for months in some animals. However, in ancient Leptospira infections, the antibody levels could be too low to be detected, therefore, even in samples from animals with clinical suspicion of leptospirosis; ancient serological reactions could not be demonstrable with MAT. The MAT protocol used in this study considered serum dilutions from $1: 100$ to $1: 1600$, consequently, it is possible that samples with titres lower than 1:100 were not detected.

The vaccine status of the animal should be considered in the interpretation of serological tests because MAT and the rapid test reacted positive in some vaccinated dogs. However, out of 15 vaccinated animals, only 3 were serological reactors to MAT (Table 2). Regarding this, since antibody titers associated with vaccination against Leptospira decrease to undetectable levels over time (Bolin 1996), the susceptibility to the infection cannot be determined with serologic tests (Martin et al. 2014). Furthermore, the absence of correlation between antibody titers after vaccination and the protection against leptospiral infection has been reported in different studies (Klaasen et al. 2003, Minke et al. 2009).

We are aware that the limitations of this study are the relatively small sample size, the low frequency of animals with clinical suspicion of leptospirosis and the unavailability of paired serum samples. However, the fact that $37.1 \%$ of dogs with no apparent clinical evidence of leptospirosis tested positive in MAT indicates the importance of informing all dog owners about the risks of leptospirosis and the need for prevention. In addition, veterinarian practitioners in southern Chile should be aware of the potential severity of leptospirosis. Seropositive dogs without clinical signs could be infected and shed the pathogen in their urine. Whether the seropositive dogs had residual antibodies due to past exposure or are indeed infected need to be treated and must be investigated.

\section{CONCLUSIONS}

The present study indicates that domestic dogs with anti-Leptospira antibodies are present in southern Chile. The most frequent serovars detected with MAT were L. interrogans serovar Canicola, followed by L. Borgpetersenii serovar Ballum and L. interrogans serovars Pomona, Autumnalis, Icterohaemorragiae and Hardjo.

The seropositivity to different serovars have implications in prevention and control of leptospirosis and in the future development of vaccines.

The immunochromatography test showed a low Se and a high SP relative to MAT as screening assay under the conditions of this study and none of the dogs with suspicion of leptospirosis, according their clinical signs, tested positive for specific IgM antibodies with this test.

Rapid and user-friendly diagnostic procedures for canine leptospirosis such as this immunochromatography assay could be important tools to use in clinical practice, but further studies are needed to obtain a more comprehensive information about their diagnostic utility, considering that diagnostic tests could not have the same performance in different geographic locations, clinical and epidemiological contexts of Leptospira infection.

Conflict of interest statement.- The authors have no competing interests.

\section{REFERENCES}

Abdoel T.H., Houwers D.J., Van Dongen A.M., Adesiyun A.A., Jiménez-Coello M., Cardoso M., Suepaul S.M., Ortega-Pacheco A. \& Smits H. 2011. Rapid test for serodiagnosis of acute canine leptospirosis. Vet. Microbiol. 150:211-213.

Acha P. \& Szyfres B. 2003. Leptospirosis, p.175-186. In: Acha P. \& Szyfres B. (Eds), Zoonosis y Enfermedades Transmisibles Comunes al Hombre y a los Animales. OPS/OMS, Washington, DC.

Alton G., Berke O., Reid-Smith R., Ojkic D. \& Prescott J.E. 2009. Increase in seroprevalence of canine leptospirosis in Ontario 1998-2006. Can. J. Vet. Res. 73:167-175.

Bharti A., Nally J., Ricaldi J., Mathias M., Lovett M., Levett P., Gilman R., Willing M., Gotuzzo E. \& Vinetz J. 2003. Leptospirosis: a zoonotic disease of global importance. Lancet Infect. Dis. 3:757-71.

Bolin C.A. 1996. Diagnosis of leptospirosis: a reemerging disease of companion animals. Semin. Vet. Med. Surg. 11:166-171.

Bolin C.A. 2000. Leptospirosis, p.185-200. In: Brownand C. \& Bolin C. (Eds), Emerging Diseases of Animals. ASM Press, Washington, DC.

Damborg P., Broens E.M., Chomel B.B., Guentherx S., Pasmansk F., Wagenaar J.A., Weese J.S., Wielerx L.S., Windahl U., Vanrompay D. \& Guardabassi 
L. 2015. Bacterial zoonoses transmitted by household pets: state of the art and future perspectives for targeted research and policy actions. J. Comp. Pathol. doi: 10.1016/j.jcpa.2015.03.004

Dey S., Madhan-Mohan C., Ramadass R. \& Nachimuthu K. 2007. Recombinant antigen-based dipstick ELISA for the diagnosis of leptospirosis in dogs. Vet. Rec. 160:186-188.

Faine S. 1994. Leptospira and Leptospirosis. CRC Press, Florida, p.229241.

Gaschen F. 2008. Canine leptospirosis. Proc. 33rd World Small Animal Veterinary Congress, Dublin, Ireland. Available online <http://www.ivis. org/proceedings/wsava/2008/lecture14/89.pdf?LA=1> Accessed on 29 Oct. 2015.

Greene C.E., Sykes J.E., Brown C.A. \& Hartmann K. 2006. Leptospirosis, p.402-417. In: Greene C.E. (Ed.), Infectious Diseases of the Dog and Cat, Saunders Elsevier, St Louis, Missouri.

Greiner M. \& Gardner I. 2000. Epidemiologic validation of veterinary diagnostic test. Prev. Vet. Med. 45:3-22.

Guerra M. 2009. Leptospirosis. J. Am. Vet. Med. Assoc. 234:472-477.

Hartskeerl R., Collares-Pereira M. \& Ellis A. 2011. Emergence, control and re-emerging of leptospirosis: dynamics of infection in the changing world. Clin. Microbiol. Infect.17:494-501.

Hartskeerl R. 2003. Leptospirose: een onderschatte infectieziekte. Ned. Tijdschr. Med. Microbiol. 11:94-98.

Klaasen H.L., Molkenboer M.L., Vrijenhoek M.P. \& Kaashoek M.L. 2003. Duration of immunity in dogs vaccinated against leptospirosis with a bivalent inactivated vaccine. Vet. Microbiol. 95:121-132.

Langston C. \& Heuter K. 2003. Leptospirosis, a re-emerging zoonotic disease. Vet. Clin. Small Anim. 33:791-807.

Lelu M., Muñoz-Zanzi C., Higgins B. \& Galloway R. 2015. Seroepidemiology of leptospirosis in dogs from rural and slum communities of Los Rios Region, Chile. BMC Vet. Res. 11:31.

Major A., Schweighauser A. \& Francey T. 2014. Increasing incidence of canine leptospirosis in Switzerland. Int. J. Environ. Res. Publ. Health 11:7242-7260.

Martin L.E., Wiggans K.T., Wennogle S.A., Curtis K., Chandrashekar R. \& Lappin L.R. 2014. Vaccine-associated Leptospira antibodies in client-owned dogs. J. Vet. Intern. Med. 28:789-792.
Minke J.M., Bey R., Tronel J.P., Latour S., Colombet G., Yvorel J., Cariou C., Guiot A.L., Cozette V. \& Guigal P.M. 2009. Onset and duration of protective immunity against clinical disease and renal carriage in dogs provided by a bi-valent inactivated leptospirosis vaccine. Vet. Microbiol. 137:137145.

Picardeau M. 2013. Diagnosis and epidemiology of leptospirosis. Med. Maladies Infect. 43:1-9.

Riedemann S. \& Zamora J. 1987. Leptospirosis animal. Serogrupos y serovares presentes en Chile y su importancia. Arch. Med. Vet. 19:69-72.

Safiullah S., Abu Saleh A. \& Munwar S. 2009. Laboratory methods for diagnosing leptospirosis: a review. Bangladesh J. Med. Microbiol. 3:39-43.

Schuller S., Francey T., Hartmann K., Hugonnard M., Kohn B., Nally J.E. \& Sykes J. 2015. European consensus statement on leptospirosis in dogs and cats. J. Small Anim. Pract. 56:169-179.

Sessions J. \& Greene C. 2004. Canine leptospirosis: epidemiology, pathogenesis and diagnosis. Comp. Cont. Educ. Pract. Vet. 26:606-618.

Silva R.F. \& Riedemann S. 2007. Frequency of canine leptospirosis in dogs attending veterinary practices determined through Microscopic Agglutination Test and comparison with isolation and immunofluorescence techniques. Arch. Med. Vet. 39:269-274.

Sykes J., Hartmann K., Lund K., Moore G., Stoddard R. \& Goldstein R. 2011. 2010 ACVIM small animal consensus statement on leptospirosis: diagnosis, epidemiology, treatment and prevention. J. Vet. Intern. Med. 25:113.

Terpstra W., Ligthart G. \& Schoone J. 1985. ELISA for the detection of specific IgM and IgG in human leptospirosis. J. Gen. Microbiol. 131:377-385.

Van de Maele I., Claus A., Haesebrouk F. \& Daminet F. 2008. Leptospirosis in dogs: a review with emphasis on clinical aspects. Vet. Rec. 163:409413.

Vieira M., Gamma-Simoes M. \& Collares-Pereira M. 2006. Human leptospirosis in Portugal: a retrospective study of eighteen years. Int. J. Infect. Dis. 10:378-386.

WHO 2001. Fatal leptospirosis, Azores Island. Wkly. Epidemiol. Rec. 76: 109-111.

WHO 2003. Human Leptospirosis: guidance for diagnosis, surveillance and control, International Leptospirosis Society (ILS). World Health Organization, Geneva, Switzerland. 